Article

\title{
Christopher Alexander's Theory of Wholeness as a Tetrad of Creative Activity: The Examples of $A$ New Theory of Urban Design and The Nature of Order
}

\author{
David Seamon $\mathbb{D}$ \\ Department of Architecture, Kansas State University, Manhattan, KS 66506, USA; triad@ksu.edu
}

Received: 1 March 2019; Accepted: 17 April 2019; Published: 21 April 2019

check for updates

\begin{abstract}
To identify and evaluate architect Christopher Alexander's theory of wholeness, this article draws on the work of British philosopher J.G. Bennett, who developed a conceptual method-what he called systematics - to clarify phenomena by drawing upon the qualitative significance of number. A central assumption of systematics is that there is something inherent in number itself that is fundamental to the way the world is and the way we can understand it. For Bennett, each whole number provides different but complementary modes for examining any phenomenon; thus, one-ness relates to the wholeness of the phenomenon; two-ness, to complementarity; three-ness, to relatedness, and so forth. This article draws on Bennett's interpretation of four-ness, summarized by a diamond-shaped symbol that he called the tetrad. Bennett claimed that the tetrad provides an interpretive means for understanding any activity directed toward a focused outcome, for example, writing a book, designing a building, or planning a new city district. The tetrad is used in this article to probe and evaluate Alexander's conceptual and practical efforts to recognize and fabricate wholeness, drawing on evidence from his Nature of Order and New Theory of Urban Design. The article first discusses the tetrad broadly and then considers how it helps to clarify Alexander's efforts to understand and make wholeness.
\end{abstract}

Keywords: Christopher Alexander; wholeness; urban design; pattern language; tetrad; systems; J.G. Bennett

\section{Introduction}

Since the late 1960s, architect Christopher Alexander has sought to understand and make a particular manner of order that he calls wholeness, which, whether in natural or human-made settings, is the "source of coherence in any part of the world" [1] (p. 90). Alexander contends that, when actualized appropriately, wholeness offers a sense of harmony that "fills and touches us" [1] (p. 15). More so, wherever there is wholeness, there is life, which involves such qualities as good health (e.g., a flourishing wetland), enjoyment (e.g., a robust urban neighborhood), handsomeness (e.g., a well-crafted door), or beauty (e.g., an elegant glazed bowl, a fine oil painting, or a splendid soaring cathedral). Alexander's first book-length efforts to understand wholeness were The Timeless Way of Building (1979), his earliest extended explication and justification of wholeness [2]; and A Pattern Language (1977), a co-authored work that identified specific designable qualities of the built environment that contribute to both human and place wholeness [3]. Alexander called these designable qualities "patterns", of which he and his research associates identified and explicated over 250, for example, "identifiable neighborhoods", "main gateways", high places", "small public squares", "intimacy gradient", "windows overlooking life", and "balconies at least six feet wide".

In the early 2000s, Alexander published his four-volume masterwork, The Nature of Order, which delineates a comprehensive means for identifying and making wholeness, whether in art, decorative 
objects, buildings, or larger-scale environments [1,4-6]. His design process incorporates two parts. First, as summarized in Table 1, he identified a set of 15 geometric properties that he claimed reoccur in all things, buildings, places, and situations that evoke wholeness, beauty, and life [1] (pp. 141-242). For example, "contrast" refers to Alexander's contention that any part of a geometric pattern is strengthened to the degree that its features are different from the features of other parts of that geometric pattern. Similarly, "local symmetries" presupposes that any larger geometrical pattern is more coherent and whole if it incorporates smaller geometric patterns arranged in symmetrical groups. Yet again, "levels of scale" argues that, for a particular geometric structure, its scalar gradations must not be too great; ideally, no more in size than half any larger structures or no more in size than twice any smaller structures. Of these 15 properties, the most important is strong centers, which refer to any manner of spatial concentration, organized focus, or place of more intense pattern or activity, for example, a well-proportioned carpet pattern, a handsomely crafted window, or an elegant building entry in which all the architectural parts interrelate in an appropriate, harmonious placement [1] (pp. 151-157).

Table 1. Alexander's 15 properties of wholeness [1] (pp. 141-242).

\begin{tabular}{lll}
\hline \multicolumn{3}{c}{ Alexander's Fifteen Structural Properties of Wholeness } \\
\hline 1. Levels of scale & 6. Good shape & 11. Roughness \\
2. Strong centers & 7. Local symmetries & 12. Echoes \\
3. Boundaries & 8. Deep interlock and ambiguity & 13. The void \\
4. Alternating repetition & 9. Contrast & 14. Simplicity and inner calm \\
5. Positive space & 10. Gradients & 15. Non-separateness \\
\hline
\end{tabular}

As illustrated in Table 2, a second integral part of Alexander's design process is a set of 10 structure-enhancing actions that establish a practical means for fabricating wholeness through an incremental intensification at all levels of environmental and physical scale, from city and urban district through building layout and massing to architectural structure, ornament, and furnishings [4] (p. 225). These actions relate both to qualities of good physical design (e.g., making centers and envisioning an integrated geometric order) and also to design qualities that sustain and strengthen human worlds (e.g., recognizing the needs of clients and users via the method of pattern language). Alexander argued that, when these 10 actions work rightly, they facilitate a design process that:

always starts from the wholeness as it currently exists at that moment... At the next moment, we take a new step-introducing one new bit of structure (always composed of new, living centers) into the whole. The new structure we introduce may be large, medium, or tiny; it may be physical or abstract; it may occur on the land itself or in a person's mind, or in the collective understanding of a group of people. But the point is that at every stage of every life-creating process, the new bit of structure which is injected to transform and further differentiate the previously existing wholeness, will always extend, enhance, and intensify the structure of the previous wholeness by creating further and stronger living centers ... The structure-enhancing step, which again and again intensifies one center and creates "hooks" to other new centers, might even be called the fundamental process. [4] (p. 216) 
Table 2. Alexander's 10 structure-enhancing actions [4] (p. 225).

\begin{tabular}{ll}
\hline \multicolumn{1}{c}{ Alexander's Ten Structure-Enhancing Actions } \\
\hline 1. Making use of step-by-step adaptation. & $\begin{array}{l}\text { 6. Working to understand the needs of clients and } \\
\text { users. }\end{array}$ \\
$\begin{array}{ll}\text { 2. Evoking and being guided by a deep feeling of the } \\
\text { whing each step to enhance the whole. }\end{array}$ & $\begin{array}{l}\text { 8. Finding coherent geometric order. } \\
\text { 3. Always making centers. }\end{array}$ \\
$\begin{array}{ll}\text { 9. Establishing a form language that rises from and } \\
\text { shapes the thing being made. }\end{array}$ \\
$\begin{array}{ll}\text { 5. Creating uniqueness everywhere. Always striving for simplicity by which the thing } \\
\text { becomes more coherent and pure. }\end{array}$ \\
\hline
\end{tabular}

Because Alexander's argument for wholeness in The Nature of Order is complex and multifaceted, I aim in this article to delineate a simplified but comprehensive portrayal of its conceptual and practical thrust, drawing partly on Nature of Order but relying predominantly on Alexander's $A$ New Theory of Urban Design, published in 1987 [7]. I use this approach because Nature of Order is so wide-ranging in its theoretical and practical parts that, ironically, one can readily lose sight of the work as a whole. By focusing on one of Alexander's most pivotal real-world concerns and professional efforts-remaking a strong sense of place in the American city-one can perhaps more readily locate the central dimensions of Alexander's theory of wholeness and better understand what wholeness is both conceptually and pragmatically.

\section{J. G. Bennett's Systematics and the Tetrad}

To identify and evaluate Alexander's theory of wholeness, I draw on the work of British philosopher J.G. Bennett (1897-1974), who developed a conceptual method — what he called systematics - to clarify phenomena by drawing upon the qualitative significance of number [8,9]. In devising an intellectual method for identifying the underlying pattern and structure of a particular "thing" - be it an object, person, group, action, relationship, situation, or process-Bennett turned to the experienced quality of whole numbers. He argued, for example, that the qualitative meaning of one-ness relates to the particular whole with which one is concerned, while the qualitative meaning of two-ness relates to the various differences, polarities, and complementarities present in that whole. Yet again, Bennett drew on three-ness to define relationship and reconciliation; four-ness, to define ordered activity; five-ness, to define significance and potential; and so forth [9] (pp. 8-17, pp. 96-99). The central assumption of systematics is that there is something inherent in number itself that is fundamental to the way the world is and the way we can understand it. Bennett's systematics has recently gained the attention of researchers in systems theory because the approach offers an innovative method for thinking about specific systems from contrasting but complementary perspectives [10] (pp. 177-178) [11] (pp. x-xi, pp. 368-370, p. 394) [12] (pp. 32-42) [13-15]. As systems scientist Martin Zwick explains, the philosophical framework of systematics "reflects deep insight" and provides for "flexibility and generativity" in better understanding any phenomenon, be it a thing, person, group, process, activity, situation, or event [15] p. 732, p. 748.

In describing the method of systematics, Bennett used the word system to designate the underlying pattern that a specific number represents. He defined a system as "a set of independent but mutually relevant terms", where term refers to "those elements of the system that express a specific character, such as universality, complementarity, dynamism, activity, potential, and so forth" [9] (p. 13). By using the Greek word for the particular number followed by the suffix - $a d$, he gave each system a name. The monad relates to one-ness and wholeness; the dyad, two-ness and complementarity; the triad, three-ness and relationship; the tetrad, four-ness and activity; and so forth. Bennett argued that each of these systems offers varying but equally accurate perspectives on the particular thing in which the researcher is interested. In this way, he or she might gain a more comprehensive and integrated 
understanding of phenomena and be better able to appreciate and to work with them. Table 3 summarizes Bennett's first six systems and their associated terms and attributes.

Table 3. The first six systems of Bennett's systematics [9] (pp. 12-14, p. 111).

\begin{tabular}{|c|c|c|}
\hline \multicolumn{3}{|c|}{ First Six Systems of Bennett's Systematics } \\
\hline System & Term & Systemic Attribute \\
\hline 1: monad & totality & wholeness \\
\hline 2: dyad & natures & difference \\
\hline 3: triad & impulses & relatedness \\
\hline 4: tetrad & sources & organized activity \\
\hline 5: pentad & limits & potential \\
\hline 6: hexad & laws & event as coalescence \\
\hline
\end{tabular}

In this article, I consider Alexander's work as an "organized activity", an aspect of human life that Bennett associated with four-ness and the tetrad. Bennett argued that the tetrad helps one to understand any sort of creative, directed process that aims for some focused outcome, for example, conducting research, writing a book, directing a play, designing a building, or planning a new urban district. Here, I describe the tetrad broadly and then consider how its structure helps clarify Alexander's efforts to understand and make wholeness.

\section{The Tetrad and Its Four Sources}

Bennett conceptualized the tetrad as the cross-filled diamond illustrated in Figure 1. Bennett claimed that the four-ness of the tetrad helps to answer the question: "What is happening and why?" and thus lays out the aspects of any intentional, coordinated activity, which in Alexander's case relates most broadly to making as a process of creating order, coherence, and life, whether one fabricates an object, building, place, or some other made thing [9] (pp. 63-76). As indicated in Figure 1, Bennett called the four points of the tetrad sources, designated by the words ground, agent, instrument, and goal. The ground is the formless, raw material that the activity aims to transform; the goal is the ideal result or end product this transformation process aims to actualize. In turn, the agent refers to the forces and qualities that drive the actualization process, and the instrument relates to the set of tools, instructions, and methods whereby the agent accomplishes the actualization practically.

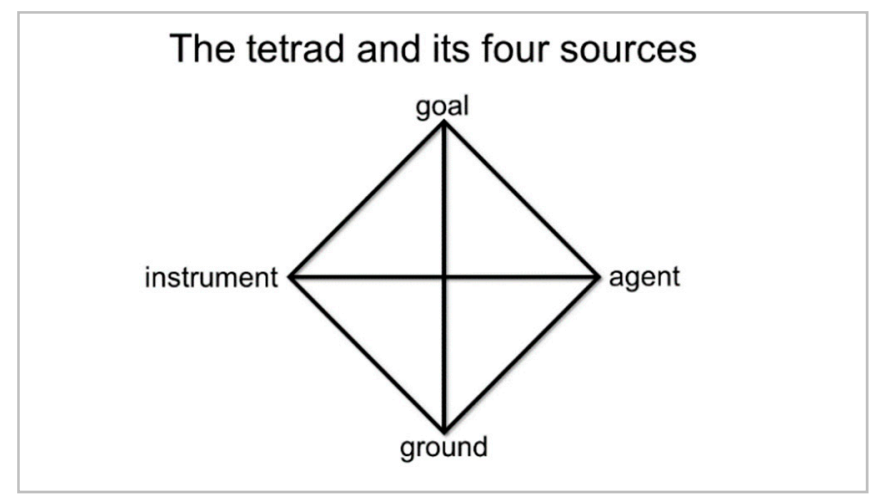

Figure 1. The tetrad and its four sources [9] (pp. 64-67).

In studying the tetrad of Figure 1, one realizes that the vertical line connecting ground and goal is related to the "ends" and outcome of the activity. In other words, the need for the ground to become more ordered and complete is present ideally in the goal, which in turn provides the vision and aspiration for an actualization of greater order potentially possible for the ground. In turn, the tetrad's horizontal line signifies the conceptual and practical "means" whereby the transformation from ground to goal might unfold through the particular knowledge, skills, and practices of the parties 
involved (agent) as they draw on some set of guidelines, procedures, and tools to actualize the process temporally (instrument).

A simple example of the tetrad is the ordered activity of cooking a meal, illustrated in Figure 2. Ideally, the aim, starting with raw ingredients and cooking facilities (ground), is an enjoyable dining experience (goal), achieved via a cook (agent) making use of a recipe and a suitable set of utensils, appliances, and cooking devices (instrument). Though cooking is an activity basic to human life, one notes potential variations in each of the four sources, for example, quality of the raw ingredients, degree of order in the kitchen, relative experience of the cook, suitability of the recipe, and demands of the dining occasion. Real-world constraints like these all contribute to whether the meal will be inedible, ordinary, or a refined gastronomic experience. For the highly skilled chef, the activity of cooking becomes so honed and secondhand that he or she may spurn recipes and make exceptional food preparation seem effortless. In contrast, a beginning cook may have so little experience that, even though he or she aims for a tasty meal, the result is barely passable.

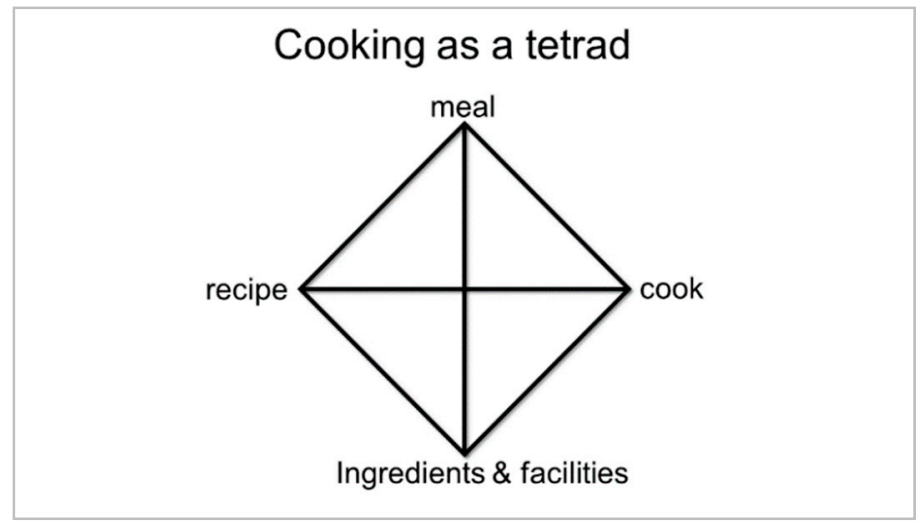

Figure 2. Cooking as a tetrad [9] (pp. 63-65).

In short, each of the four sources and their relationships can vary widely with the result that the particular organized activity succeeds or fails; if the activity is accomplished, the quality of that accomplishment may be exceptional, adequate, or minimally acceptable. Envisioning an activity via the tetrad allows one to locate the factors whereby possibilities might become actual and to evaluate those factors as they potentially contribute to or undermine the quality of the activity's outcome. I next identify the tetrad that underlies the urban-design process that Alexander develops in New Theory of Design. I then evaluate the four sources of this tetrad and their interrelationships. I end by drawing on this tetrad to clarify the much more complex design process that Alexander delineates in Nature of Order.

\section{Alexander's Process of Urban Place-Making}

To illustrate the application of Bennett's tetrad to Alexander's theory of wholeness, I turn to his New Theory of Urban Design, an effort to suggest a way of revitalizing the American city, which Alexander pictures as chaotic, dehumanizing, and placeless [7] (pp. 1-16, p. 234). In this book, Alexander offers seven rules, as he calls them, which he argues might provide a healing action for the city and lead to a renewed sense of urban place. These rules are summarized in Table 4. For example, rule 1 - "piecemeal growth" — states that the best construction increments are small; thus, in any attempt to strengthen the city or its districts, there should be an even mix of small, medium, and large construction projects. Building on rule 1, rule 2- "the growth of larger wholes" - directs how specific design projects should be envisioned as integral parts of a larger urban fabric. This rule requires that "every building increment must be chosen, placed, planned, formed, and given its details in such a way as to increase the number of wholes that exist in space" [7] (p. 248). 
Table 4. Alexander's seven rules for urban design [7] (pp. 31-99).

\begin{tabular}{l} 
Alexander's Seven Rules for Urban Design \\
\hline 1. Piecemeal growth \\
2. The growth of larger wholes \\
3. Visions \\
4. Positive outdoor space \\
5. Layout of buildings \\
6. Construction details \\
7. Formation of centers \\
\hline
\end{tabular}

Of these seven rules, the most pivotal is the last-formation of centers—which, beginning with New Theory, becomes Alexander's primary conceptual and practical means for clarifying and extending his earlier "pattern language" ideas. As explained above, a center relates to spatial concentration and any place of more intense pattern and activity, for example, a well-placed kiosk, a graceful arcade, a welcoming building, a lively plaza full of people enjoying themselves, or an entire city neighborhood that is well-liked and cared for [1] (pp. 151-157) [7] (pp. 72-99). Whatever its particular nature and scale, a center is a region of more intense physical and experiential order that provides for the relatedness of things, people, situations, and events. In this sense, the strongest centers, city-wise, gather the urban parts in a relationship of belonging, including city dwellers. Further, wherever one finds life and wholeness in the city, centers are never alone but mutually implicated and intermeshed at many levels of scale: "The wholeness of any portion of the world is the system of larger and smaller centers, in their connections and overlap" [1] (pp. 90-91).

Alexander illustrates the use of his seven rules through a simulation experiment conducted with architecture graduate students at the University of California at Berkeley in a design studio taught by him and colleagues Ingrid King and Howard Davis. The 19 students focused on 30 acres of the San Francisco waterfront just north of the Bay Bridge destined for development (see Figure 3). The major task was to transform these 30 acres, mostly empty, into a district of buildings, streets, plazas, and parks that would all contribute to a sense of life, atmosphere, and wholeness. Eventually, the students converted the waterfront site into a set of places that included a pedestrian mall, a main square, a waterfront park, a market, and fishing pier (see Figure 3). 


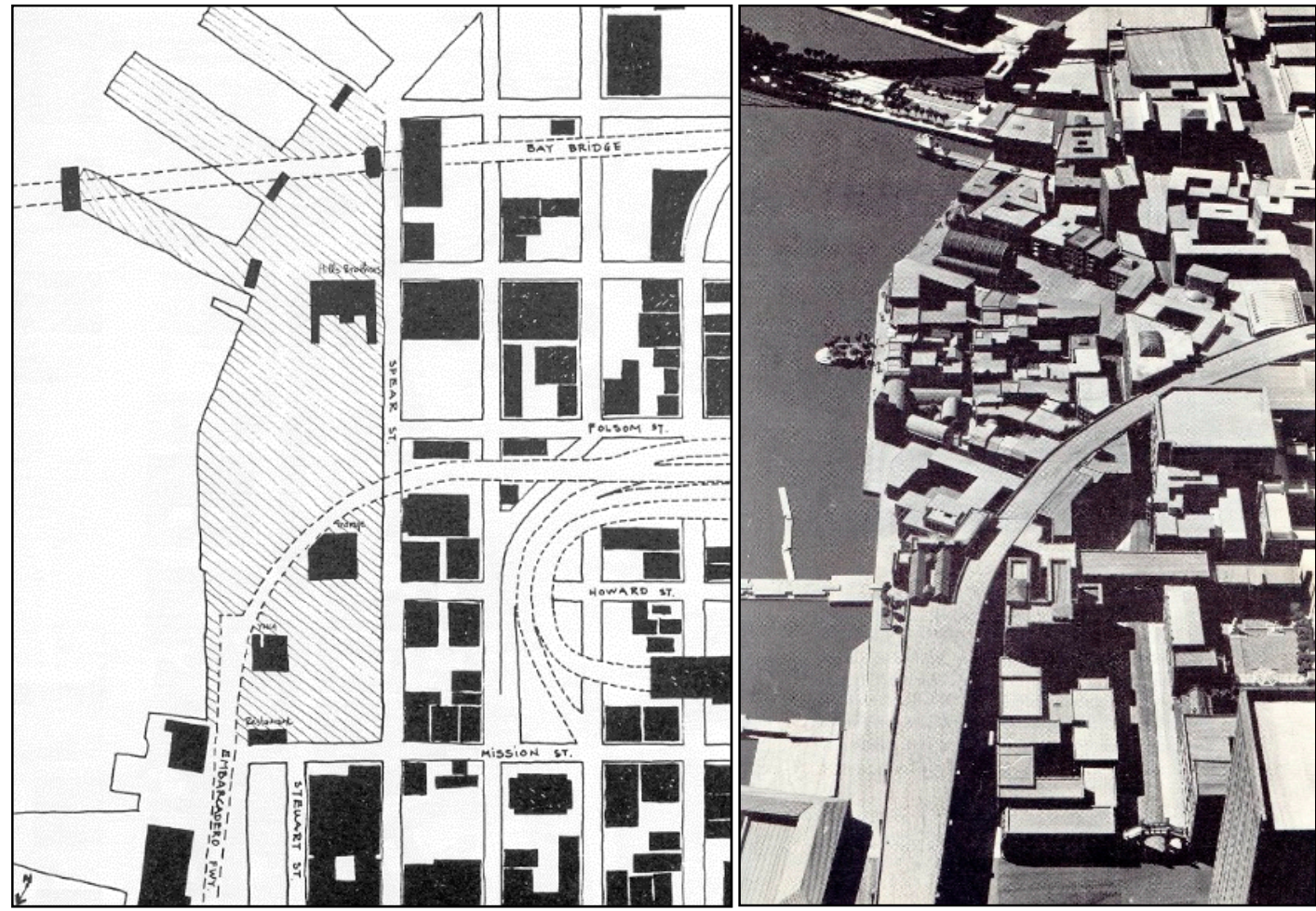

Figure 3. Left: San Francisco waterfront, with the site to be developed marked by hatched lines; Right: photograph of a wooden model of the San Francisco waterfront project after 89 projects; copyright 1987 Christopher Alexander and used with permission [7] (p. 104, p. 107).

As the main means for developing this design scheme, Alexander asked the students to imagine themselves as developers, designers, and representatives of community groups. In turn, Alexander, King, and Davis took the role of an evaluation committee responsible for guiding the growth process. No student's design idea (what Alexander calls, in rule 3, "vision") could be finalized until the committee had evaluated the idea and considered strengths and weaknesses. All faculty and students were involved in all discussions about every project, so at least theoretically there was much mutual understanding as to the project's progress and ultimate aims [7] (pp. 108-110).

\section{New Theory of Urban Design as Tetrad}

How can Alexander's theory of urban design be understood in light of Bennett's tetrad? As shown in Figure 4, I call this tetrad, "Alexander's Theory of Urban Design", and identify its four sources as site, wholeness, rules, and dialogue. The ground of Alexander's design process is the 30-acre site, which incorporates minimal order and is in need of development. The goal for the site is wholeness, which for the waterfront district relates to coherence, robustness, and a unique sense of place. The instrument for actualizing wholeness is Alexander's seven rules, through which the participants in the design process gain understanding and the waterfront gains realization. These rules are set in motion through the agent of students and teachers, who role-play a developer/committee relationship founded in dialogue, in other words, continual group discussion and awareness of who is planning and designing what, where, and when; and how each increment potentially contributes to the wholeness of the site and its urban sense of place. 


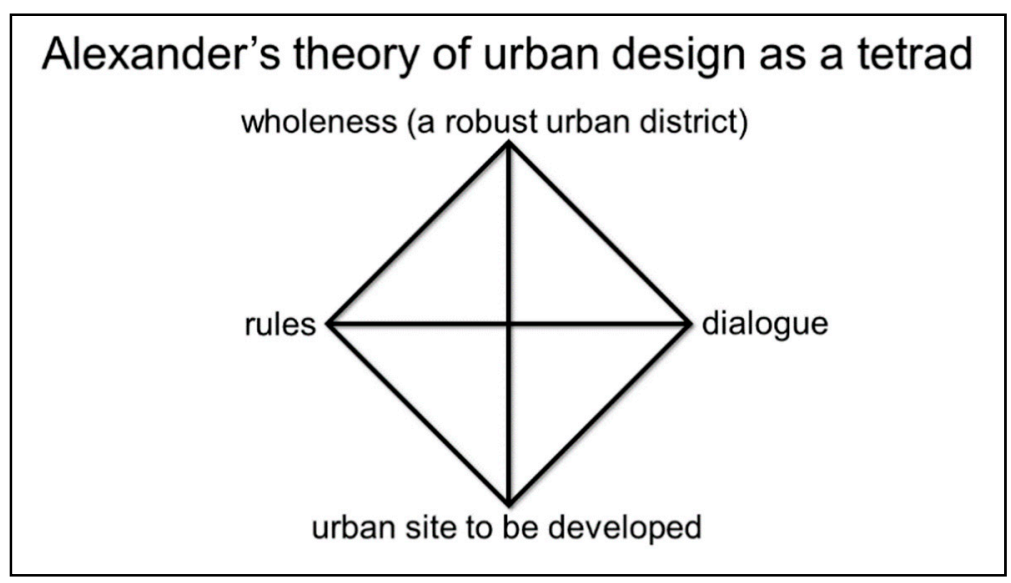

Figure 4. Alexander's theory of urban design as a tetrad.

How well does Alexander's approach to urban design work? Bennett argued that one way to consider this question is to examine the six links joining the tetrad's four sources. These links, Bennett claimed, indicate that the four sources "are not separate but interconnected and influencing each other" [9] (p. 69) [14] pp. 731-734). First, there is the lower-left link between site and rules, which speaks to the prospect that development will maintain the site's basic integrity even as the rules give guidance to ways in which that basic integrity can be enhanced and made more coherent and alive. Second, there is the lower-right link between site and dialogue. In Alexander's approach, the prospect here is that the students' unrefined perceptions of the site are given creative formation through a growing awareness of what the site is and how, in the unfolding stages of the design process, the site has been transformed so far.

Does the final waterfront design maintain the integrity of the San Francisco site yet use that integrity as the basis for creative design, drawing on the potential of rules and dialogue? One must answer both yes and no. Aspects of the design that seem to respect the site include the pedestrian mall and the many increments-for example, the fishing pier, the waterfront park, and the boat-repair area-that draw on the proximity of the site to water. On the other hand, there are increments that seem less in character with the site, for example, the buildings along the expressway (how to deal with noise and pollution?) and the grid of streets (is a rectilinear pattern most appropriate for the site's waterfront character?). There is also the larger practical question of whether the site would be economically viable with the land uses proposed in the waterfront design.

Turning to the upper half of the tetrad, one can consider, first, the upper-left link between rules and wholeness, which relates to the transformation of the rules into an actual environment with a strong urban character. Second, there is the upper-right link between dialogue and wholeness, which relates to the faculty and students' participation in the design process and their collective success in evoking order, rightness, and place identity for the site. How well did the rules transcribe themselves into an actual sense of place wholeness? Again, there is both success and failure. On one hand, as compared to the arbitrariness of most conventional urban planning and design, the waterfront scheme is superior in that it incorporates a sense of human scale and relaxed informality. On the other hand, the final design is weak in terms of individual buildings that often lack a sense of unity and have the appearance of "a late nineteenth-century pseudo-renaissance style of architecture" [7] (p. 235). In spite of these criticisms, the completed design does evoke a certain crispness and feeling of innovation; one senses that, with some projects, the students experienced instances of genuine insight and vision sympathetic to the site. In this sense, there were moments in the design process when the relationship between dialogue and wholeness worked in a real and powerful way, a situation that offers much hope for design education and practice modeled after Alexander's approach.

Finally, there are the tetrad's vertical and horizontal links between site/wholeness and rules/dialogue. As already indicated, the link between site and wholeness relates to the realization 
of the site as a more coherent, robust place, while the link between rules and dialogue relates to the growing understanding of students and faculty, especially in relation to how the rules might be refined to make the design process more effective in generating wholeness. One of the most encouraging aspects of Alexander's experiment is that designs over time appear to have improved as the students had continuing practice with the seven rules and were able to work with them fluidly and creatively. As Alexander explains,

The students who took part [in this studio] were ... involved in all discussions about every project. As a result, there came about a considerable amount of unspoken coherence in the project, which arose from people's mutual understanding of what we were trying to accomplish ... In the last stages of development, the students were able to function almost entirely without the guidance from the committee, since the seven rules had been completely absorbed and understood. [7] (pp. 109-110)

At the same time, the simulation refined Alexander's own sense of the design process and led him to emphasize, in the evaluation of the experiment in the book's final section, that the rules regarding wholeness need further refinement so that increments are more in touch with the larger whole and, therefore, have a more organic, unself-conscious quality. Throughout the book, Alexander emphasizes that he is not entirely satisfied with these rules. He says that they are not formulated precisely enough with the result that the waterfront design is "too much an aggregate of parts, not a single well-formed whole" [7] (p. 237).

\section{Intensifying Wholeness?}

Because of his emphasis on wholeness, the most significant question about Alexander's approach relates to the line in the tetrad linking site and wholeness. Did the waterfront site experience healing, and was it transformed into a place of considerable order and life? The final design is laudable in its incorporating a functional intricacy and diversity not typically found in conventional urban planning. As Alexander writes, the design expresses an "organic, personal, and human character which we associate with many of the most beautiful cities of the past, and which seems to be responsible for their success as environments. And it clearly does not have the obsessive, dead character of most 'urban design' projects of recent decades" [7] (p. 234).

At the same time, Alexander admits that the design has several weaknesses, the greatest of which is that the project does not have a strong enough sense of order at the largest scale [7] (pp. 236-238). He is particularly dissatisfied with the main square, since it was to be the heart of an integrated site. He points out that the main square and its immediate surroundings offer some sense of order, but this order does not project itself into the other parts of the site. He concludes that, if the theory were perfect, it would not only have produced a main square somewhere near the center but also a

field-like gradient, which could be felt throughout the project, in which every part of the project would "point" towards the main square. Thus, somehow, the whole project would have a physical structure showing a kind of centralized gradient, all of it sloping (metaphorically) toward the middle. [7] (p. 237)

If we now turn to the link in the tetrad between agent and instrument, we face the controversial question of whether Alexander's theory of urban design could ever be used successfully in actual city place-making, in short, its social, economic, and political practicality. How could Alexander's approach to urban design ever be implemented in a real-world community of residents, businesspeople, politicians, administrators, and general public, whose criteria for success vary greatly and are often much different from the quest for wholeness that is Alexander's central aim? He makes this dilemma a strength by pointing out that his emphasis on wholeness leads to "an entirely different effect from current practice in urban design and goes far to remedy the defects which cities have today" [7] (pp. 248-24). His hope is that a committed, funded community with the necessary material, economic, 
and psychological resources might someday apply his approach to envision and regenerate exuberant urban places.

\section{The Nature of Order as a Tetrad}

To conclude, I ask whether the tetrad I have delineated here for the less complicated context of New Theory might be generalized to the more complex, sophisticated theory of Nature of Order. In thinking through that work as a tetrad, I arrive at the four sources illustrated in Figure 5: ground as materials and situation from which the made thing arises; instrument as the 15 structural properties; agent as the 10 structure-enhancing actions of maker and client (if there is a client); and goal as life-giving order and wholeness.

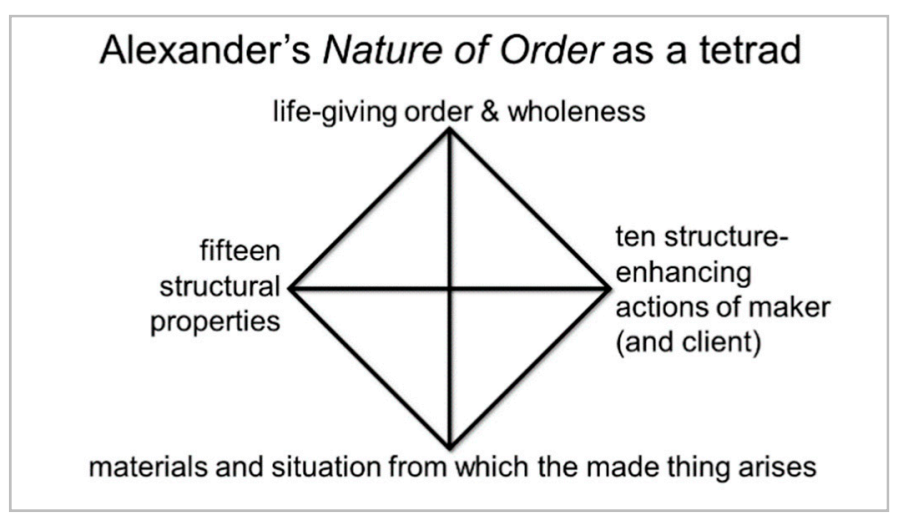

Figure 5. Alexander's Nature of Order as a tetrad.

In one sense, three of the four volumes of Nature of Order can be interpreted as laying out three of the four sources of this tetrad: volume 1 deals largely with an explication of the 15 structural properties (indicated by the volume's title The Phenomenon of Life); volume 2 lays out the 10 structure-enhancing actions (indicated by the volume's title The Process of Creating Life); and volume 4 points toward the ontological and metaphysical grounds of life-giving order and wholeness (indicated by the volume's title The Luminous Ground). Volume 3 might be said to illustrate all four of the tetrad's sources in that Alexander demonstrates how he uses the generative process laid out in volumes 1 and 2 to create specific life-enhancing things, buildings, and places.

As I suggested at the start of this article, perhaps the greatest weakness of Nature of Order is its attempt to be both in-depth and comprehensive: in-depth, in terms of defining a set of actions that work for any process of making, whether creating an art work, decorative object, building, or place; comprehensive, in terms of providing evidence of made things at all levels of environmental and architectural scale-from neighborhood and public spaces through buildings, rooms, and construction and ornamental details-even works of art and decorative art. On one hand, it is not really clear whether the 15 structural properties have the power to facilitate, at such a broad range of material scale, the clear understanding offered by Alexander's earlier pattern language or the seven rules of New Theory. Another concern is that, like the unfocused nature of the dialogue process in New Theory, the 10 structure-enhancing actions may not always have the practicality or resilience to move the making process in such a way that it evolves toward the life-giving order and wholeness that Alexander cherishes. On the other hand, one could argue that the depth and comprehensiveness of Nature of Order is its strength in that it demonstrates-at least with partial success - that any kind of material creating is essentially the same: facilitating a multi-staged making that is grounded in a shared understanding of basic principles and properties and leads to a fabrication that evokes wellbeing, belonging, wholeness, and life.

In this regard, I ask whether the built work Alexander presents in volume 3 of Nature of Order manifests the life-sustaining wholeness that he advocates. Broadly, the answer is both yes and no. Some of his projects, especially many of the houses, seem awkward, roughly constructed, and second-rate. 
On the other hand, there are a good number of buildings and other constructed projects that, in various ways, evoke the sense of clarity, dignity, and life for which Alexander strives. One impressive example is England's West Dean Visitor's Centre, a building that, both inside and out, appears to be striking architecture, expressing a placid dignity shaped by careful site placement; a simple, imposing form; and tastefully integrated materials, including brick, stone, and concrete [5] (pp. 16-17, pp. 145-147) [6] (pp. 118-129). Another persuasive example is a self-help housing project in Mexacali, Mexico, in which five low-income families designed and built their own homes, following many of the principles delineated in Nature of Order [16,17]. Perhaps most convincing is the 36-building, 10-million-dollar Eishin School, a 2000-student combination high school and college in suburban Tokyo, Japan, begun in 1981 and largely completed by 1990 [18,19]. These three projects are telling examples of what a life-enhancing architecture might be and demonstrate that Alexander's approach to making can have effective, practical results and is feasible for a wide range of situations, clients, and building types.

Ultimately, however, Alexander would not wish to be judged on his finished projects alone. Rather, he sees each of these projects as an experiment: as another opportunity to figure out and refine a way of learning and making that can generate order and life. Here lies Alexander's unique contribution: his ceaseless effort to understand the design-build process and to identify principles, procedures, and actions that generate things, environments, and places that are coherent, robust, and empowering.

\section{Conclusions}

Alexander's oeuvre has been interpreted and evaluated in a wide range of ways, both positive and negative [20-36]. I have argued here that J.G. Bennett's explication of the tetrad provides one conceptual means to clarify and evaluate Alexander's theory of wholeness and to demonstrate that it offers one integrated, comprehensive means for facilitating effective, creative designs and fabrications. During his professional life, Alexander has produced a remarkable body of work, which most succinctly can be described as the development of conceptual and practical ways to understand, to design, and to make wholeness. Throughout his many writings, he has presented wholeness in different ways, and I have argued here that interpreting his work via J.G. Bennett's tetrad allows one to see an underlying continuity both in terms of successful design process as well as conceptual and practical means for assuring successful design outcomes.

Many architects and urban designers have a clear vision of what they wish to accomplish design-wise but offer no clear method or process for achieving that vision. In this regard, Alexander is an invaluable exception because he provides a thoughtful explication of what good design is and how it might be actualized generatively. If Bennett is correct in contending that any organized activity directed toward a focused outcome must necessarily incorporate the tetradic structure of ground, goal, direction, and instrument, then Alexander's theory of understanding and making wholeness is comprehensive in its parts, providing for a practical implementation of these four tetradic sources. Perhaps what is most encouraging about Alexander's design process is that it incorporates both agent (the 10 structure-enhancing actions) and instrument (the 15 structural properties). In short, the generation of wholeness out of the original, incomplete ground is provided agency, impetus, and structure via "wholeness-extending transformations" potentially actualized by the 10 structure-enhancing actions as they incorporate the 15 structural properties [37] (pp. 14-15). Obviously, Alexander's method of design is not easy, quick, clear-cut, or guaranteed. It requires an intensive, longstanding personal and professional commitment to producing buildings and places that evoke an exuberance, elegance, and life-enhancing ambience. Alexander argues, however, that such engaged, abiding dedication is integral to good design and fabrication. He claims that the built environment will remain uninspired, alienating, and placeless until designers return to a "timeless way of building" that has "generated the greatly loved and now treasured traditional environments throughout the world" [37] (p. 15).

Alexander is unique as an architect and architectural thinker in that he has pondered the design process carefully and developed an ensemble of means-pattern languages, geometric properties, wholeness-extending transformations, and so forth—to actualize environmental and place wholeness. 
Though perhaps not all his built projects facilitate the overarching quality of integration, grace, and life for which he aims, his work is trailblazing in that his steady, wide-ranging efforts mark a fruitful, organized path that might help others to envision and make better buildings, places, and environments.

Funding: This research received no external funding.

Conflicts of Interest: The author declares no conflict of interest.

\section{References}

1. Alexander, C. The Nature of Order. Vol. 1: The Phenomenon of Life; Center for Environmental Structure: Berkeley, CA, USA, 2002.

2. Alexander, C. The Timeless Way of Building; Oxford University Press: New York, NY, USA, 1987.

3. Alexander, C.; Angel, S.; Fiksdahl-King, I.; Ishikawa, S.; Jacobson, M.; Silverstein, M. A Pattern Language: Towns, Buildings, Constructions; Oxford University Press: New York, NY, USA, 1977.

4. Alexander, C. The Nature of Order, Vol. 2: The Process of Creating Life; Center for Environmental Structure: Berkeley, CA, USA, 2002.

5. Alexander, C. The Nature of Order, Vol. 3: A Vision of a Living World; Center for Environmental Structure: Berkeley, CA, USA, 2005.

6. Alexander, C. The Nature of Order, Vol. 4: The Luminous Ground; Center for Environmental Structure: Berkeley, CA, USA, 2004.

7. Alexander, C.; Anninou, A.; King, I.; Neis, H. A New Theory of Urban Design; Oxford University Press: New York, NY, USA, 1987.

8. Bennett, J.G. The Dramatic Universe: Man and his Nature; Hodder \& Stoughton: London, UK, 1966.

9. Bennett, J.G. Elementary Systematics; Bennett Books: Santa Fe, NM, USA, 1993.

10. Bortoft, H. Taking Appearance Seriously; Floris Books: Edinburgh, UK, 2012.

11. Bortoft, H. The Wholeness of Nature; Floris Books: Edinburgh, UK, 1996.

12. Seamon, D. Life Takes Place: Phenomenology, Lifeworlds, and Place Making; Routledge: London, UK, 2018.

13. Wood, S. Favorite Places: Spatial and Temporal Dimensions of Place Attachment. Environ. Arch. Phenomenol. 2014, 25, 10-16.

14. Zwick, M. Freedom as a Natural Phenomenon. Found. Sci. 2017, 22, 1-10. [CrossRef]

15. Zwick, M. Ideas and Graphs: The Tetrad of Activity. Int. J. Gen. Syst. 2018, 47, 731-750. [CrossRef]

16. Alexander, C.; Davis, H.; Martinez, J.; Corner, D. The Production of Houses; Oxford University Press: New York, NY, USA, 1985.

17. Alexander, C.; Fromm, D.; Bosselman, P. Mexacali Revisited: Seven Years Later. Places 1984, 1, 76-91.

18. Alexander, C.; Neis, H.; Moore Alexander, M. The Battle for the Life and Beauty of the Earth; Oxford University Press: New York, NY, USA, 2012.

19. Guttmann, E.; Kaiser, G.; Mazanek, C. Shifting Patterns: Christopher Alexander und der Eishin Campus; Park Books: Zurich, Switzerland, 2017.

20. Bhatt, R. Christopher Alexander's Pattern Language: An Alternative Exploration of Space-Making Practices. J. Arch. 2010, 15, 711-729. [CrossRef]

21. Coates, G.; Seamon, D. Promoting a Foundational Ecology Practically Through Christopher Alexander's Pattern Language: The Example of Meadowcreek. In Dwelling, Seeing and Designing: Toward a Phenomenol-ogical Ecology; Seamon, D., Ed.; State University of New York Press: Albany, New York, USA, 1993; pp. 331-355.

22. Dawes, M.J.; Ostwald, M.J. Christopher Alexander's A Pattern Language: Analysing, Mapping and Classifying the Critical Response. City Territ. Arch. 2019, 3, 1-14. [CrossRef]

23. Grabow, S. Christopher Alexander and the Search for a New Paradigm in Architecture; Oriel Press: London, UK, 1983.

24. Jiang, B. A Topological Representation for Taking Cities as a Coherent Whole. Geogr. Anal. 2018, 50, 298-313. [CrossRef]

25. Jiang, B. A Complex-Network Perspective on Alexander's Wholeness. Phys. A Stat. Mech. Appl. 2016, 463, 475-484. [CrossRef]

26. Kemmis, D. The Good City and the Good Life; Houghton Mifflin: New York, NY, USA, 1995. 
27. Kohn, W. The Lost Prophet of Architecture. Wilson Q. 2002, 26-34.

28. Mehaffy, M.W. Generative Methods in Urban Design: Christopher Alexander and his Continuing Influence. Urb. Des. Int. 2007, 12, 41-49. [CrossRef]

29. Park, Y. The Network of Patterns: Creating a Design Guide Using Christopher Alexander's Pattern Language. Environ. Plan. B Plan. Des. 2015, 42, 593-614. [CrossRef]

30. Pursuit of a Living Architecture: Continuing Christopher Alexander's Quest for a Humane and Sustainable Building Culture; Pontikis, K.; Rofe, Y. (Eds.) Common Ground Publishing: Champaign, IL, USA, 2016.

31. Salingaros, N.A. The Structure of Pattern Languages. Arch. Res. Q. 2000, 4, 149-161. [CrossRef]

32. Saunders, W. Commentary: Ever more Popular, ever more Dogmatic. Arch. Rec. 2002, 190, 93-96.

33. Seamon, D. Grasping the Dynamism of Urban Place: Contributions from the Work of Christopher Alexander, Bill Hillier, and Daniel Kemmis. In Reanimating Places; Tom, M., Ed.; Ashgate: Burlington, VT, USA, 2004; pp. 123-145.

34. Seamon, D. Making a New World [review of Christopher Alexander, The Nature of Order, vols. 2-4]. Trad. Build. 2005, 186-188.

35. Seamon, D. Christopher Alexander and a Phenomenology of Wholeness. In Pursuit of a Living Architecture: Continuing Christopher Alexander's Quest for a Humane and Sustainable Building Culture; Pontikis, K., Rofe, Y., Eds.; Common Ground Publishing: Champaign, IL, USA, 2016; pp. 50-66.

36. Week, D. Patterns with Positivism: Alexander Reinterpreted for the 21st Century. In Pursuit of a Living Architecture: Continuing Christopher Alexander's Quest for a Humane and Sustainable Building Culture; Pontikis, K., Rofe, Y., Eds.; Common Ground Publishing: Champaign, IL, USA, 2016; pp. 67-98.

37. Alexander, C. Empirical Findings from the Nature of Order. Environ. Arch. Phenomenol. 2007, 18, 11-19.

(C) 2019 by the author. Licensee MDPI, Basel, Switzerland. This article is an open access article distributed under the terms and conditions of the Creative Commons Attribution (CC BY) license (http://creativecommons.org/licenses/by/4.0/). 